\title{
Existence and Uniqueness of Periodic Solutions for Second Order Differential Equations
}

\author{
Yuanhong Wei \\ College of Mathematics, Jilin University, Changchun 130012, China \\ Correspondence should be addressed to Yuanhong Wei; yhwei@amss.ac.cn \\ Received 12 June 2014; Accepted 16 August 2014; Published 27 August 2014 \\ Academic Editor: Mohamed Abdalla Darwish
}

Copyright (C) 2014 Yuanhong Wei. This is an open access article distributed under the Creative Commons Attribution License, which permits unrestricted use, distribution, and reproduction in any medium, provided the original work is properly cited.

We study some second order ordinary differential equations. We establish the existence and uniqueness in some appropriate function space. By using Schauder's fixed-point theorem, new results on the existence and uniqueness of periodic solutions are obtained.

\section{Introduction}

In this paper, we are concerned with the existence and uniqueness of periodic solution for the nonlinear equation

$$
x^{\prime \prime}=f\left(t, x, x^{\prime}\right),
$$

where $f: \mathbb{R} \times \mathbb{R} \times \mathbb{R} \rightarrow \mathbb{R}$ is continuous, periodic in $t$ with period $T$, and $C^{1}$ with respect to $\left(x, x^{\prime}\right)$.

Because of wide interests in physics and engineering, periodic solutions of second order differential equations have been investigated by many authors. We refer the reader to [17] and the references cited therein.

The purpose of this paper is to study the existence and uniqueness of periodic solution in some appropriate function space $C_{T}^{1}$. To be precise, we first derive a result of existence and uniqueness, when the nonlinearity is a $C^{1}$ function with respect to $\left(x, x^{\prime}\right)$. Then, a similar result concerning the existence of periodic solutions is obtained, when the nonlinearity is not a $C^{1}$ function.

Throughout this paper we use the following assumptions.

(A1) There exist two continuous functions $a(t)$ and $b(t)$ such that, for all $t \in[0, T]$,

$$
0 \nsupseteq a(t) \leqslant f_{x}\left(t, x, x^{\prime}\right) \leqslant b(t),
$$

where $0 \leqq a(t)$ means $0 \leqslant a(t)$ and $0 \not a(t)$.
(A2) There exists a positive constant $M$ such that, for all $t \in[0, T]$,

$$
\left|f_{x^{\prime}}\left(t, x, x^{\prime}\right)\right| \leqslant M
$$

We now present our main results of this paper.

Theorem 1. Let assumptions (A1) and (A2) hold. Then (1) has a unique T-periodic solution.

Remark 2. We point out that the condition $0 \lesseqgtr a(t) \leqslant f_{x}$ in (A1) is necessary. Let us take the following equation, for example:

$$
x^{\prime \prime}=C \text {, }
$$

where $C$ is a constant and $C \neq 0$. The equation has no periodic solution.

Remark 3. Consider the following example:

$$
x^{\prime \prime}=\sin t \cdot \sin x \cdot \sin x^{\prime}+x .
$$

We can easily check that assumptions (A1) and (A2) hold. By Theorem 1 , the equation has a unique $2 \pi$-periodic solution.

We also consider the case when the right-hand side of the equation is only continuous. In this case, we establish the existence of periodic solutions for the differential equation

$$
x^{\prime \prime}=f\left(t, x, x^{\prime}\right) x+g\left(t, x, x^{\prime}\right),
$$


where $f, g: \mathbb{R} \times \mathbb{R} \times \mathbb{R} \rightarrow \mathbb{R}$ are continuous and periodic in $t$ with period $T$. We assume the following.

(A3) There exist continuous functions $a(t)$ and $b(t)$ and a positive constant $M$ such that

$$
\begin{gathered}
0 \supsetneqq a(t) \leqslant f\left(t, x, x^{\prime}\right) \leqslant b(t), \\
\left|g\left(t, x, x^{\prime}\right)\right| \leqslant M,
\end{gathered}
$$

for all $t \in[0, T]$.

Then, we have the following result.

Theorem 4. Let (A3) hold. Then (6) has at least one T-periodic solution.

Remark 5. Consider the following example:

$$
x^{\prime \prime}=2 x+x \sin x^{\prime}+\cos t \cdot \cos x .
$$

By Theorem 4 , the equation has at least one $2 \pi$-periodic solution.

To establish the main results, we introduce some appropriate function space. By using Schauder's fixed-point theorem, the existence and uniqueness of periodic solutions are obtained. We know from the anonymous referee that the proof of this paper can be simplified, by using the results in [8]. The proof of this paper can be seen as an application of Schauder's fixed-point theorem.

\section{Preliminary}

In this section, We first introduce the function space, in which we will obtain the periodic solution for the problem. Then, some preliminary lemma is introduced, which is valuable for the proof of our main results.

Let $C_{T}^{1}$ be the space of continuously differentiable $T$ periodic functions, with norm $\|\cdot\|$ given by

$$
\|x\|=\max _{t \in[0, T]}|x(t)|+\max _{t \in[0, T]}\left|x^{\prime}(t)\right| .
$$

It is well known that $C_{T}^{1}$ is a Banach space.

We now introduce the following lemma.

Lemma 6. $u(t)$ and $v(t)$ are T-periodic functions. If $0 \lessgtr v(t)$, for all $t \in R$, then the equation

$$
x^{\prime \prime}=u(t) x^{\prime}+v(t) x
$$

has a unique T-periodic solution $x(t) \equiv 0$.

Proof. Assume there exists a T-periodic solution $x(t)$, $x(t) \not \equiv$. Since $0 \lessgtr v(t), x(t)$ is not a constant. Otherwise we will get a contradiction by substituting $x=C \neq 0$ into (10). Then we claim that there exist $t_{0}$ and $t_{1}, t_{0}<t_{1}$, such that

$$
\begin{aligned}
& x(t)>0, \quad \text { for } t \in\left(t_{0}, t_{1}\right) ; \\
& x^{\prime}\left(t_{0}\right)>0, \quad x^{\prime}\left(t_{1}\right)=0 .
\end{aligned}
$$

Now we prove it.
Case 1. $x(t)$ has zero points. Assume that $x(\theta)=0$; then, we have $x^{\prime}(\theta) \neq 0$ because the initial value problem of $(10)$ has a unique solution. If $x^{\prime}(\theta)>0$, then we let $t_{0}=\theta$; if $x^{\prime}(\theta)<0$, by the periodic condition, there exists $t_{0}, t_{0}>\theta$, such that

$$
x(t)<0, \quad t \in\left(\theta, t_{0}\right) ; \quad x\left(t_{0}\right)=0 .
$$

So $x^{\prime}\left(t_{0}\right)>0$. Also by the periodic condition there exists $\eta$, $\eta>t_{0}$, such that

$$
x(t)>0, \quad t \in\left(t_{0}, \eta\right) ; \quad x(\eta)=0 .
$$

So we can find a $t_{1} \in\left(t_{0}, \eta\right)$ such that $x^{\prime}\left(t_{1}\right)=0$. Thus, under Case 1 we verify (11) holds.

Case 2. $x(t)$ has no zero point. Then $x(t)$ has a constant sign. Suppose $x(t)>0$ (if not we can consider the $T$-periodic solution $\bar{x}(t)=-x(t), \bar{x}(t)>0)$. Since $x(t)$ is periodic and is not a constant, there exists $t_{0}$ such that $x^{\prime}\left(t_{0}\right)>0$, $x\left(t_{0}\right)=x\left(t_{0}+T\right)$. So we can find a $t_{1}, t_{1}>t_{0}, x^{\prime}\left(t_{1}\right)=0$. Then we prove that (11) holds.

Multiplying both sides of (10) by $\exp \left\{-\int_{t_{0}}^{t_{1}} u(s) d s\right\}$ and integrating between $t_{0}$ and $t_{1}$ we get

$$
0>-x^{\prime}\left(t_{0}\right)=\int_{t_{0}}^{t_{1}} v(t) x(t) \exp \left\{-\int_{t_{0}}^{t_{1}} u(s) d s\right\} d t \geqslant 0,
$$

which leads to a contradiction. This completes the proof of Lemma 6.

Remark 7. We can also prove the following. If $u(0)=u(T)$, $v(0)=v(T), 0 \lessgtr v(t)$, a.e. $t \in[0, T]$, then the following periodic boundary value problem,

$$
\begin{aligned}
& x^{\prime \prime}=u(t) x^{\prime}+v(t) x, \quad t \in[0, T], \\
& x(0)=x(T), \quad x^{\prime}(0)=x^{\prime}(T),
\end{aligned}
$$

also has a unique solution $x(t) \equiv 0$.

\section{Proof of the Main Results}

Proof of Theorem 1. First prove the uniqueness. Assume that $x_{1}(t)$ and $x_{2}(t)$ are two $T$-periodic solutions of (1). Setting

$$
x(t)=x_{1}(t)-x_{2}(t),
$$

we get

$$
\begin{gathered}
x(t+T)=x(t), \\
x^{\prime \prime}=f\left(t, x_{1}, x_{1}^{\prime}\right)-f\left(t, x_{2}, x_{2}^{\prime}\right) \\
=f_{x}\left(t, x_{1}+\theta_{1}\left(x_{1}-x_{2}\right), x_{1}^{\prime}\right) x \\
+f_{x^{\prime}}\left(t, x_{2}, x_{2}+\theta_{2}\left(x_{1}-x_{2}\right)\right) x^{\prime} .
\end{gathered}
$$

From assumption (A1) we know

$$
0 \lessgtr a(t) \leqslant f_{x}\left(t, x_{1}+\theta_{1}\left(x_{1}-x_{2}\right), x_{1}^{\prime}\right) .
$$

Hence, by Lemma $6, x(t) \equiv 0$. 

form:

We next prove the existence. Rewrite (1) in the following

$$
\begin{aligned}
x^{\prime \prime}= & f\left(t, x, x^{\prime}\right) \\
= & \left(f\left(t, x, x^{\prime}\right)-f(t, x, 0)\right) \\
& +(f(t, x, 0)-f(t, 0,0))+f(t, 0,0) \\
= & \int_{0}^{1} f_{x^{\prime}}\left(t, x, \theta_{1} x^{\prime}\right) d \theta_{1} x^{\prime} \\
& +\int_{0}^{1} f_{x}\left(t, \theta_{2} x, 0\right) d \theta_{2} x+f(t, 0,0) .
\end{aligned}
$$

From Lemma 6, for each $x \in C_{T}^{1}$, the equation

$$
\begin{aligned}
y^{\prime \prime}= & \int_{0}^{1} f_{x^{\prime}}\left(t, x, \theta_{1} x^{\prime}\right) d \theta_{1} y^{\prime} \\
& +\int_{0}^{1} f_{x}\left(t, \theta_{2} x, 0\right) d \theta_{2} y+f(t, 0,0)
\end{aligned}
$$

has a unique $T$-periodic solution because the corresponding homogeneous equation only has trivial $T$-periodic solution $x(t) \equiv 0$.

We define operator

$$
P: C_{T}^{1} \longrightarrow C_{T}^{1}
$$

for given $x \in C_{T}^{1}, y(t)=P[x](t)$ is the unique $T$-periodic solution of (20). Then the existence of the $T$-periodic solution is equivalent to the existence of fixed point of $P$ in the space $C_{T}^{1}$. We will prove that $P$ is continuous and compact, and $P\left(C_{T}^{1}\right)$ is a bounded subset of $C_{T}^{1}$.

Proof of Continuity. For any convergent sequence $\left\{x_{k}\right\} \subset C_{T}^{1}$, satisfying $x_{k} \rightarrow x_{0}(k \rightarrow \infty)$, let $y_{k}=P x_{k}$. Then

$$
\begin{aligned}
y_{k}^{\prime \prime}= & \int_{0}^{1} f_{x^{\prime}}\left(t, x_{k}, \theta_{1} x_{k}^{\prime}\right) d \theta_{1} y_{k}^{\prime} \\
& +\int_{0}^{1} f_{x}\left(t, \theta_{2} x_{k}, 0\right) d \theta_{2} y_{k}+f(t, 0,0) .
\end{aligned}
$$

We claim that $\left\{y_{k}\right\}$ is a bounded sequence in $C_{T}^{1}$. If not, we can find a subsequence of $\left\{y_{k}\right\}$ (for convenience we also use the same notations) such that $\left\|y_{k}\right\| \rightarrow \infty(k \rightarrow \infty)$. Let $\omega_{k}=y_{k} /\left\|y_{k}\right\|$. Then $\left\{\omega_{k}\right\} \subset C_{T}^{1},\left\|\omega_{k}\right\|=1$,

$$
\begin{aligned}
\omega_{k}^{\prime \prime}= & \int_{0}^{1} f_{x^{\prime}}\left(t, x_{k}, \theta_{1} x_{k}^{\prime}\right) d \theta_{1} \omega_{k}^{\prime} \\
& +\int_{0}^{1} f_{x}\left(t, \theta_{2} x_{k}, 0\right) d \theta_{2} \omega_{k}+\frac{f(t, 0,0)}{\left\|y_{k}\right\|} .
\end{aligned}
$$

So $\left\|\omega_{k}^{\prime \prime}\right\| \leqslant M+\max _{t \in[0, T]} b(t)+1<\infty(k \rightarrow \infty)$. Since $\left\{\omega_{k}^{\prime \prime}\right\}$ is bounded and

$$
\omega_{k}^{\prime}(t)=\omega_{k}^{\prime}(0)+\int_{0}^{t} \omega_{k}^{\prime \prime}(s) d s,
$$

$\left\{\omega_{k}^{\prime}\right\}$ is bounded and equicontinuous sequence of functions. Furthermore, by

$$
\omega_{k}(t)=\omega_{k}(0)+\int_{0}^{t} \omega_{k}^{\prime}(s) d s,
$$

$\left\{\omega_{k}\right\}$ is bounded and equicontinuous sequence of functions. By Ascoli-Arzelà Theorem, $\left\{\omega_{k}^{\prime}\right\}$ and $\left\{\omega_{k}\right\}$ contain a uniformly convergent subsequence, respectively, (for convenience we also use the same notations) such that

$$
\omega_{k} \stackrel{1}{\longrightarrow} \omega_{0}, \quad \omega_{k}^{\prime} \stackrel{1}{\longrightarrow} v_{0},
$$

where the notation “ $\stackrel{1}{\rightarrow}$ " means uniform convergence. Obviously, $\omega_{0}, v_{0} \in C_{T}^{1}$. From (23) and (24), we obtain

$$
\begin{aligned}
\omega_{k}^{\prime}(t)= & \omega_{k}^{\prime}(0) \\
& +\int_{0}^{t}\left(\int_{0}^{1} f_{x^{\prime}}\left(s, x_{k}, \theta_{1} x_{k}^{\prime}\right) d \theta_{1} \omega_{k}^{\prime}\right. \\
& \left.\quad+\int_{0}^{1} f_{x}\left(s, \theta_{2} x_{k}, 0\right) d \theta_{2} \omega_{k}+\frac{f(s, 0,0)}{\left\|y_{k}\right\|}\right) d s .
\end{aligned}
$$

Let $k \rightarrow \infty$. From (25) and (27), we get

$$
\begin{aligned}
& \omega_{0}(t)=\omega_{0}(0)+\int_{0}^{t} v_{0}(s) d s, \\
& v_{0}(t)=v_{0}(0)+\int_{0}^{t}\left(\int_{0}^{1} f_{x^{\prime}}\left(s, x_{k}, \theta_{1} x_{k}^{\prime}\right) d \theta_{1} v_{0}(s)\right. \\
& \left.\quad+\int_{0}^{1} f_{x}\left(s, \theta_{2} x_{k}, 0\right) d \theta_{2} \omega_{0}(s)\right) d s .
\end{aligned}
$$

Hence

$$
\omega_{0}^{\prime \prime}=\int_{0}^{1} f_{x^{\prime}}\left(t, x_{0}, \theta_{1} x_{0}^{\prime}\right) d \theta_{1} \omega_{0}^{\prime}+\int_{0}^{1} f_{x}\left(t, \theta_{2} x_{0}, 0\right) d \theta_{2} \omega_{0} .
$$

By Lemma 6, we have $\omega_{0} \equiv 0$, which is in contradiction with $\left\|\omega_{0}\right\|=1$, so $\left\{y_{k}\right\}$ is a bounded sequence. Then, by (22), we know $\left\{y_{k}^{\prime \prime}\right\}$ is bounded, so $\left\{y_{k}^{\prime}\right\}$ and $\left\{y_{k}\right\}$ are bounded and equicontinuous sequences of functions. By Ascoli-Arzelà Theorem, $\left\{y_{k}^{\prime}\right\}$ and $\left\{y_{k}\right\}$ contain a uniformly convergent subsequence, respectively, (for convenience we also use the same notations) such that

$$
y_{k} \stackrel{1}{\longrightarrow} y_{0}, \quad y_{k}^{\prime} \stackrel{1}{\longrightarrow} \bar{v}_{0} .
$$


We know

$$
\begin{aligned}
& y_{k}^{\prime}(t)=y_{k}^{\prime}(0)+\int_{0}^{t} y_{k}^{\prime \prime}(s) d s \\
& =y_{k}^{\prime}(0)+\int_{0}^{t}\left(\int_{0}^{1} f_{x^{\prime}}\left(s, x_{k}, \theta_{1} x_{k}^{\prime}\right) d \theta_{1} y_{k}^{\prime}\right. \\
& +\int_{0}^{1} f_{x}\left(s, \theta_{2} x_{k}, 0\right) d \theta_{2} y_{k} \\
& +f(s, 0,0)) d s \\
& y_{k}(t)=y_{k}(0)+\int_{0}^{t} y_{k}^{\prime}(s) d s .
\end{aligned}
$$

When $k \rightarrow \infty$, from (31), we obtain

$$
\begin{aligned}
y_{0}^{\prime \prime}= & \int_{0}^{1} f_{x^{\prime}}\left(t, x_{0}, \theta_{1} x_{0}^{\prime}\right) d \theta_{1} y_{0}^{\prime} \\
& +\int_{0}^{1} f_{x}\left(t, \theta_{2} x_{0}, 0\right) d \theta_{2} y_{0}+f(t, 0,0) .
\end{aligned}
$$

By the uniqueness we know $y_{0}=P x_{0}$; thus, operator $P$ is continuous.

Proof of Compactness. For each bounded set $S \subset C_{T}^{1}$, we claim that $P(S)$ is bounded in $C_{T}^{1}$. If not, by an analogous manner as above we will reach a contradiction. For every $x \in S$, $y=P x$ is defined by (20). Since $\left\|y^{\prime}\right\|,\|y\|,\left\|f_{x}\right\|$, and $\left\|f_{x^{\prime}}\right\|$ are all bounded, then $\left\|y^{\prime \prime}\right\|<\infty$. Proceeding as proof of continuity we conclude that $\left\{y^{\prime}\right\}$ and $\{y\}$ are bounded and equicontinuous sequences of functions. By the Ascoli-Arzelà Theorem, $P$ is a compact operator.

We claim that $P\left(C_{T}^{1}\right)$ is bounded in $C_{T}^{1}$. If not, there exist $\left\{x_{k}\right\}, k=1,2, \ldots$, such that $\left\|P x_{k}\right\| \rightarrow \infty(k \rightarrow \infty)$. Let $y_{k}=P x_{k}$. Then (22) holds. Take $\omega_{k}=y_{k} /\left\|y_{k}\right\|$. Then $\left\{\omega_{k}\right\} \subset C_{T}^{1},\left\|\omega_{k}\right\|=1$, and (23), (24), (25), and (27) hold. By the above proof we know $\left\{\omega_{k}^{\prime}\right\}$ and $\left\{\omega_{k}\right\}$ are bounded and equicontinuous sequences of functions and contain a uniformly convergent subsequence, respectively, (also use the same notations) such that

$$
\omega_{k} \stackrel{1}{\longrightarrow} \omega_{0}, \quad \omega_{k}^{\prime} \stackrel{1}{\longrightarrow} v_{0}, \quad\left\|\omega_{0}\right\|=1 .
$$

Sequences $\left\{\int_{0}^{1} f_{x^{\prime}}\left(t, x_{k}, \theta_{1} x_{k}^{\prime}\right) d \theta_{1}\right\}_{k=1}^{\infty}$ and $\left\{\int_{0}^{1} f_{x}\left(t, \theta_{2} x_{k}\right.\right.$, $\left.0) d \theta_{2}\right\}_{k=1}^{\infty}$ are bounded sequences in $L^{2}[0, T]$. By the weakly sequential compactness of $L^{2}$ space, both of them have a weakly convergent subsequence (also use the same notations) such that

$$
\begin{aligned}
& \int_{0}^{1} f_{x^{\prime}}\left(t, x_{k}, \theta_{1} x_{k}^{\prime}\right) d \theta_{1} \stackrel{w}{\longrightarrow} f_{1}(t), \\
& \int_{0}^{1} f_{x}\left(t, \theta_{2} x_{k}, 0\right) d \theta_{2} \stackrel{w}{\longrightarrow} f_{2}(t),
\end{aligned}
$$

in $L^{2}[0, T]$, where $\stackrel{w}{\longrightarrow}$ ” means weak convergence. Obviously,

$$
\begin{array}{r}
\left|f_{1}(t)\right| \leqslant M, \quad 0 \lessgtr a(t) \leqslant f_{2}(t) \leqslant b(t), \\
\text { a.e. } t \in[0, T] .
\end{array}
$$

When $k \rightarrow \infty$, from (25) and (27), for a.e. $t \in[0, T]$, we have

$$
v_{0}^{\prime}(t)=f_{1}(t) v_{0}(t)+f_{2}(t) \omega_{0}(t), \quad \omega_{0}^{\prime}(t)=v_{0}(t)
$$

Then

$$
\begin{gathered}
\omega_{0}^{\prime \prime}(t)=f_{1}(t) \omega_{0}^{\prime}(t)+f_{2}(t) \omega_{0}(t) \\
\omega_{0}(0)=\omega_{0}(T), \quad \omega_{0}^{\prime}(0)=\omega_{0}^{\prime}(T) .
\end{gathered}
$$

By Remark 3, we obtain $\omega_{0} \equiv 0$, which is in contradiction with $\left\|\omega_{0}\right\|=1$. Then there exists a constant $K>0$ such that $\|P x\| \leqslant K, x \in C_{T}^{1}$.

Assume $D=\left\{x \in C_{T}^{1},\|x\| \leqslant K+1\right\}$. By Schauder's fixedpoint theorem, $P: D \rightarrow D$ has at least one fixed point. This completes the proof of Theorem 1 .

Proof of Theorem 4. For each $x \in C_{T}^{1}$, from Lemma 6, we know the equation

$$
y^{\prime \prime}=f\left(t, x, x^{\prime}\right) y+g\left(t, x, x^{\prime}\right)
$$

has a unique $T$-periodic solution because the corresponding homogeneous equation only has trivial $T$-periodic solution $x(t) \equiv 0$.

We define $\bar{P}: C_{T}^{1} \rightarrow C_{T}^{1}$, for each given $x \in C_{T}^{1} y(t)=$ $\bar{P}[x](t)$ is the unique $T$-periodic solution of (38). Hence the existence of the periodic solutions is equivalent to the existence of fixed points of $\bar{P}$ in the Banach space $C_{T}^{1}$.

Proceeding as the proof of Theorem 1 we can prove that $\bar{P}$ is a compact continuous operator and $\bar{P}\left(C_{T}^{1}\right)$ is a bounded subset of $C_{T}^{1}$.

Then there exists a constant $K_{1}>0$ such that $\|\bar{P} x\| \leqslant K_{1}$, for all $x \in C_{T}^{1}$. Let $\bar{D}=\left\{x \in C_{T}^{1},\|x\| \leqslant K_{1}+1\right\}$. By Schauder's fixed-point theorem, $\bar{P}: \bar{D} \rightarrow \bar{D}$ has at least one fixed point. This completes the proof of Theorem 4 .

\section{Another Simple Proof}

Actually, by the anonymous referee, we know that the proof of the theorem can be much simplified if we use the theorem in [8]. In fact, for equation

$$
u^{(n)}=f\left(t, u, \ldots, u^{(n-1)}\right)
$$

where $f: \mathbb{R}^{n+1} \rightarrow \mathbb{R}$ is continuous and $T$-periodic in $t$, Theorem 2.2 of [8] implies the following propositions. 
Lemma 8. Let there exist continuous and T-periodic in the first argument functions $f_{k}: \mathbb{R}^{n+1} \rightarrow \mathbb{R}(k=1,2, \ldots, n)$ such that

$$
\begin{array}{r}
\left|f\left(t, x_{1}, \ldots, x_{n}\right)-\sum_{k=1}^{n} f_{k}\left(t, x_{1}, \ldots, x_{n}\right) x_{k}\right| \leqslant r, \\
p_{1 k}(t) \leqslant f_{k}\left(t, x_{1}, \ldots, x_{n}\right) \leqslant p_{2 k}(t) \quad(k=1,2, \ldots, n)
\end{array}
$$

are satisfied on $\mathbb{R}^{n+1}$, where $r=$ const $>0$ and $p_{i k}$ : $\mathbb{R} \rightarrow \mathbb{R}(i=1,2 ; k=1,2, \ldots, n)$ are continuous $T$-periodic functions. Let, moreover, for any continuous $T$ periodic functions $p_{k}: \mathbb{R} \rightarrow \mathbb{R}(k=1,2, \ldots, n)$, satisfying

$$
p_{1 k}(t) \leqslant p_{k}(t) \leqslant p_{2 k}(t) \quad(k=1,2, \ldots, n),
$$

the equation

$$
u^{(n)}=\sum_{k=1}^{n} p_{k}(t) u^{(k-1)}
$$

have no nontrivial T-periodic solution. Then equation has at least one T-periodic solution.

Lemma 9. Let the function $f$ in the last $n$ arguments have continuous partial derivatives, satisfying

$$
p_{1 k}(t) \leqslant \frac{\partial f_{k}\left(t, x_{1}, \ldots, x_{n}\right)}{\partial x_{k}} \leqslant p_{2 k}(t) \quad(k=1,2, \ldots, n),
$$

where $p_{i k}: \mathbb{R} \rightarrow \mathbb{R}(i=1,2 ; k=1,2, \ldots, n)$ are continuous $T$-periodic functions. Let, moreover, for any T-periodic $p_{k}$ : $\mathbb{R} \rightarrow \mathbb{R}(k=1,2, \ldots, n)$, satisfying (41), (42) has no nontrivial $T$-periodic solution. Then, the equation has a unique $T$-periodic solution.

On the basis of these theorems, we can prove the theorem by checking the conditions in the previous lemma. By Lemma 6 , the conditions above can be easily proved.

\section{Conflict of Interests}

The author declares that there is no conflict of interests regarding the publication of this paper.

\section{Acknowledgments}

This paper is supported by National Natural Science Foundation of China (Grant no. 11301209). The author would like to thank the anonymous referee for the valuable comments and suggestions on the paper, especially for the considerations in Section 4. The author would like to thank Professor Yong Li for his helpful instruction and valuable suggestions.

\section{References}

[1] F. Cong, "Periodic solutions for second order differential equations," Applied Mathematics Letters, vol. 18, no. 8, pp. 957-961, 2005.
[2] J. Ward, "Periodic solutions for a class of ordinary differential equations," Proceedings of the American Mathematical Society, vol. 78, no. 3, pp. 350-352, 1980.

[3] R. Kannan, "Existence of periodic solutions of nonlinear differential equations," Transactions of the American Mathematical Society, vol. 217, pp. 225-236, 1976.

[4] K. Schmitt, "A note on periodic solutions of second order ordinary differential equations," SIAM Journal on Applied Mathematics, vol. 21, pp. 491-494, 1971.

[5] Q. Yao, "Positive solutions of nonlinear second-order periodic boundary value problems," Applied Mathematics Letters, vol. 20, no. 5, pp. 583-590, 2007.

[6] I. Kiguradze and S. Stanck, "On periodic boundary value problem for the equation $u^{\prime \prime}=f\left(t, u, u^{\prime}\right)$ with one-sided growth restrictions on f,' Nonlinear Analysis, vol. 48, pp. 1065-1075, 2002.

[7] P. Yan and M. Zhang, "Higher order non-resonance for differential equations with singularities," Mathematical Methods in the Applied Sciences, vol. 26, no. 12, pp. 1067-1074, 2003.

[8] I. Kiguradze, "Boundary value problems for systems of ordinary differential equations," Itogi Nauki i Tekhniki. Seriya Sovremennye Problemy Matematiki. Noveishie Dostizheniya, vol. 30, pp. 3-103, 1987 (Russian), English translation Journal of Soviet Mathematics, vol. 43, no. 2, pp. 2259-2339, 1988. 


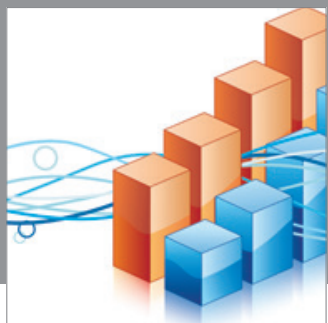

Advances in

Operations Research

mansans

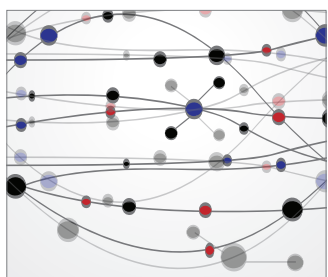

The Scientific World Journal
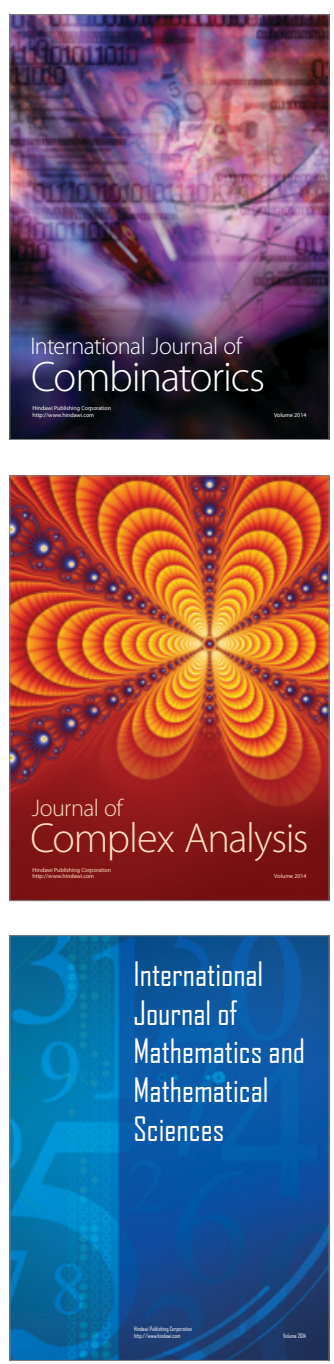
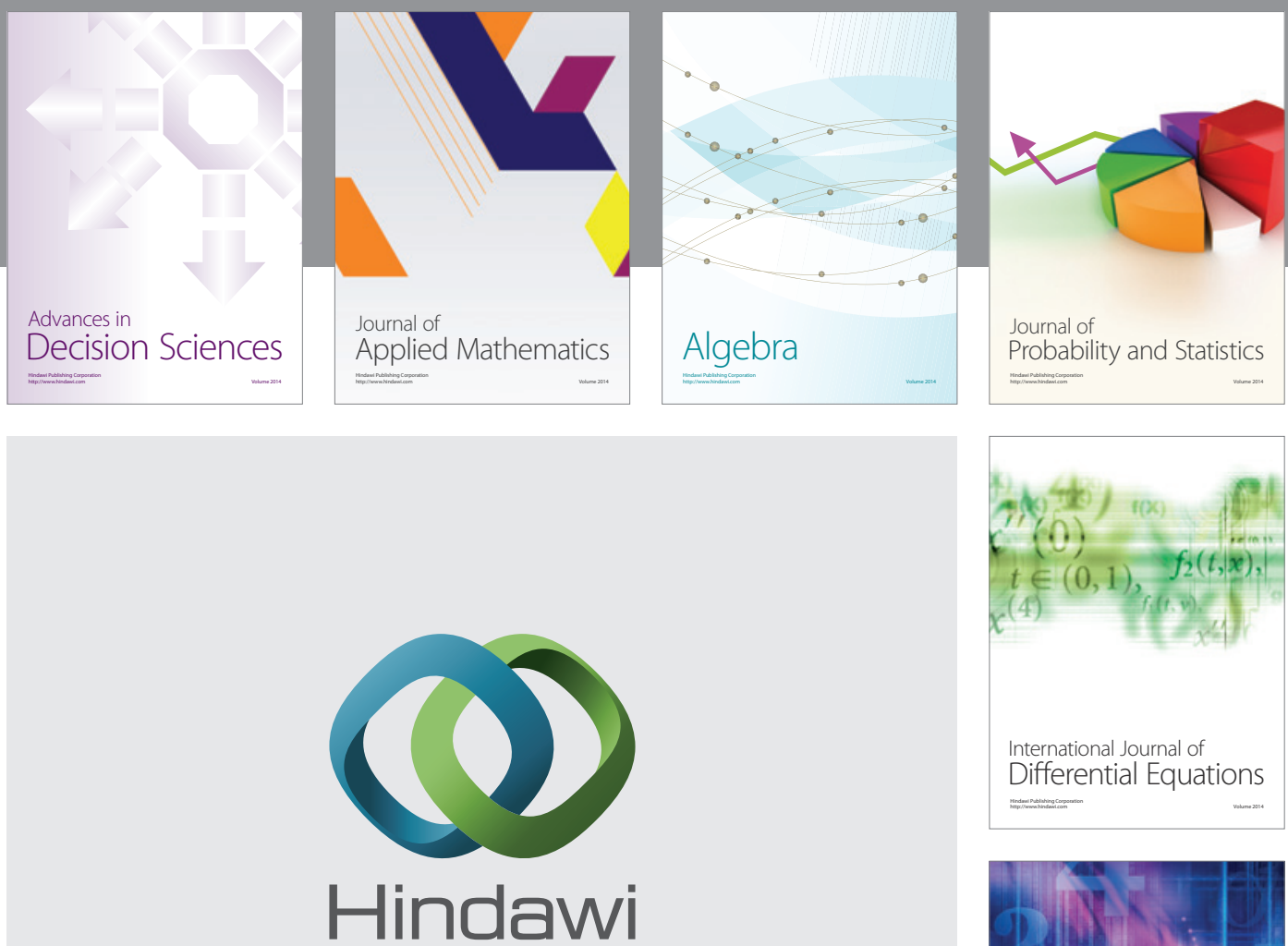

Submit your manuscripts at http://www.hindawi.com
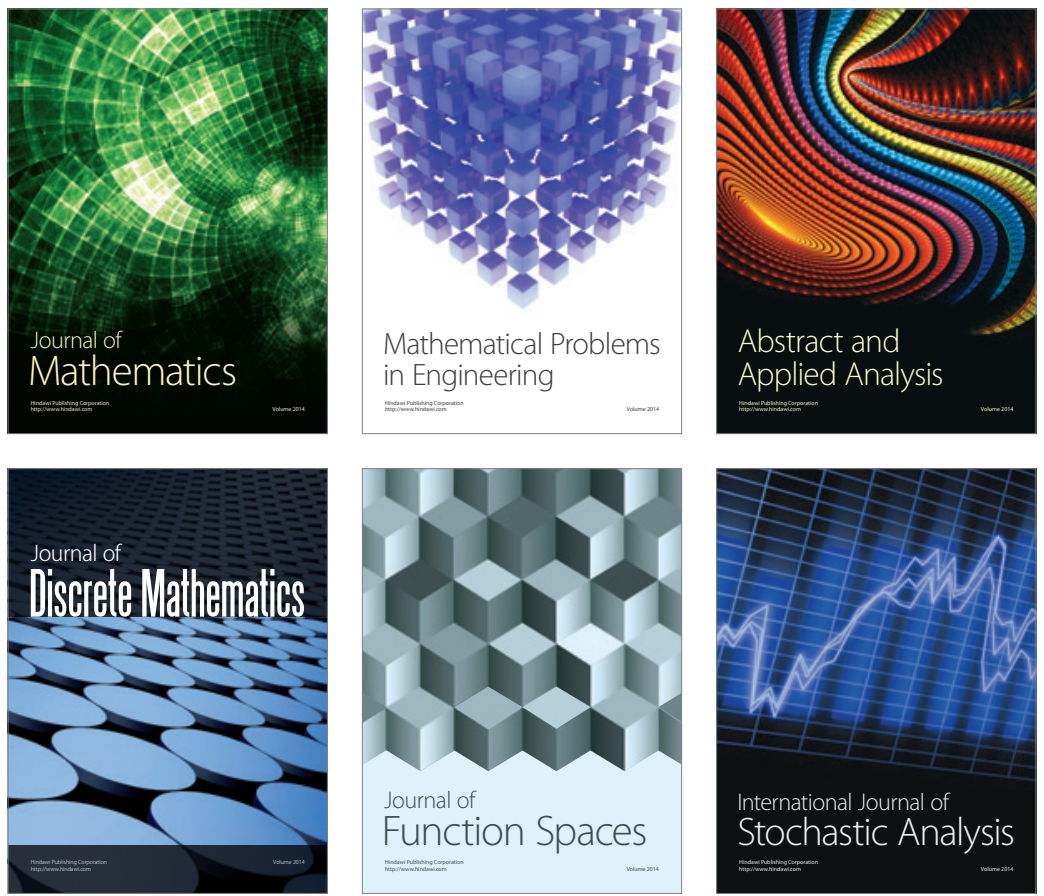

Journal of

Function Spaces

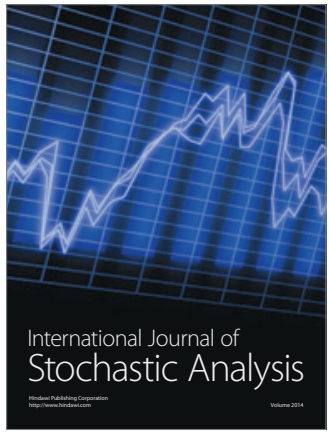

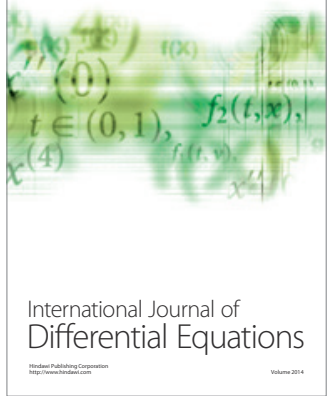
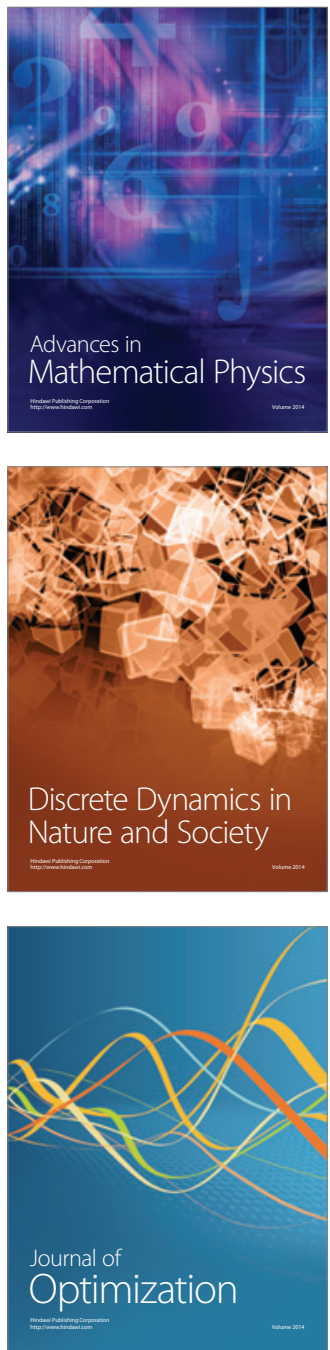